\title{
Antibiotic Sensitivity and Clinical Outcome for Methicillin Resistant Staphylococcus Aureus
}

\author{
Dennis O. Laryea*1, Yaw A. Amoako² and Joyce Asamoah ${ }^{1}$ \\ ${ }^{1}$ Public Health Unit, Komfo Anokye Teaching Hospital, Kumasi, Ghana; ${ }^{2}$ Department of Medicine, Komfo Anokye Teaching Hospital, \\ Kumasi, Ghana
}

\section{Objective}

To review laboratory and clinical data on cases of Methicillin Resistant Staphylococcus aureus infections

\section{Introduction}

Methicillin Resistant Staphylococcus Aureus (MRSA) is a strain of Staphylococcus aureus resistant to methicillin/oxacillin. The high incidence of resistance to most commonly used antibiotics including carbapenems and cephalosporins among strains of MRSA present challenges to the management of cases. Vancomycin has been identified as a common antibiotic with demonstrated activity against MRSA. In most developing countries including Ghana, MRSA is not regarded as an issue of public health importance. It is not among the priority diseases under surveillance in Ghana. Active efforts at identifying cases and instituting appropriate preventive measures are largely non-existent. This is due to the unavailability of laboratories with requisite human resource and logistics to run tests for MRSA. This has further compounded the perception of non-importance with respect to MRSA in developing countries. We therefore set out to review cases of MRSA seen in a Teaching Hospital in Ghana to estimate the burden of the infection and to examine the trends in antibiotic sensitivity and clinical outcome of cases.

\section{Methods}

We reviewed laboratory records on cases of MRSA seen at the Komfo Anokye Teaching Hospital (KATH) in Kumasi Ghana from January to December 2011 followed by a review of the clinical notes of identified cases. In KATH, Oxacillin is the antibiotic utilised in the identification of MRSA following the isolation of Staphylococcus aureus on culture of the appropriate specimen. Further antibiotic sensitivity tests are conducted based on available antibiotic discs as well as previously observed sensitivities. No molecular or genetic tests are conducted for present cases.

\section{Results}

A total of seventy four (74) cases of MRSA were identified following the laboratory record review. The majority of cases were male [42 (56.8\%)]. The Neonatal Intensive Care Unit of the Hospital accounted for $33.8 \%$ of cases. No cases were recorded in the months of January, February, March, June and July. The months of April and May recorded a case each. The months of August to December all recorded at least 10 cases each.

Blood was the commonest specimen for isolates of MRSA. A total of $64(86.5 \%)$ cases were isolated from blood. Wound swabs accounted for $9.5 \%$ of cases. The majority of isolates were sensitive to Vancomycin $(98.3 \% ; \mathrm{n}=57)$. There was also demonstrated sensitivity to Ciprofloxacin $(95.2 \% ; n=21)$ and Gentamycin $(65.2 \% ; n=23)$.

We could only trace the clinical notes of $48(64.9 \%)$ cases. Septicaemia was the most common clinical diagnosis before laboratory testing $(43.8 \% ; n=48)$ with bronchopneumonia $(8.3 \%)$ and Gastroenteritis $(8.3 \%)$ as the other common diagnoses. The majority of cases were discharged satisfactorily with deaths recorded among 6 cases (Case fatality rate of $12.6 \%$ ).

\section{Conclusions}

MRSA has the propensity to prolong hospital admission and also cause morbidity and mortality. Our review indicates a high mortality rate among confirmed cases of MRSA in Kumasi. Although a significant number of cases of MRSA were recorded in KATH during the period, no interventions were instituted as there are no protocols or guidelines in place for MRSA.

There is the need to establish a surveillance system for MRSA and develop guidelines for managing cases and potential outbreaks. There is also the need to further investigate the high numbers of cases of MRSA recorded in the Neonatal Unit. We also recommend the provision of other advanced means of diagnosis and management for MRSA.

\section{Keywords}

MRSA; Ghana; Sensitivity; Resistance

\section{Acknowledgments}

We wish to acknowledge the Staff of the Komfo Anokye Teaching Hospital Public Health Unit and the Microbiology laboratory

\section{References}

1. Warshawsky, B; Hussain, Z; Gregson, DB; Alder, R; Austin, M; Bruckschwaiger D et al Hospital- And Community-Based Surveillance of Methicillin-Resistant Staphylococcus Aureus: Previous Hospitalization is the Major Risk Factor Infection Control and Hospital Epidemiology 2000; 21(11): 724-727

2. Robicsek, A; Beaumont,J L; Paule, SM; Hacek, DM; Thomson Jr.,RB; Kaul, KL; Peggy King, RN, MBA; and Peterson, LR. Universal Surveillance for Methicillin-Resistant Staphylococcus aureus in 3 Affiliated Hospitals Annals of Internal Medicine 2008; 148 (6): 410- 418

3. Anupurba S, Sen M R, Nath G, Sharma B M, Gulati A K, Mohapatra T M. Prevalence of methicillin resistant staphylococcus aureus in a tertiary referral hospital in eastern Uttar Pradesh. Indian J Med Microbiol [serial online] 2003; 21:49-51http://www.ijmm.org/text. asp?2003/21/1/49/8318

\section{*Dennis O. Laryea \\ E-mail: denola@live.com}

